\title{
Incentive Driven Information Sharing in Delay Tolerant Mobile Networks
}

\author{
Yan Wang*, Mooi-Choo Chuah ${ }^{\dagger}$,Yingying Chen* \\ ${ }^{*}$ Dept. of ECE, Stevens Institute of Technology \\ $\dagger$ Dept. of CSE, Lehigh University \\ Castle Point on Hudson, Hoboken, NJ 07030 \\ 27 Memorial Drive West Bethlehem, PA 18015 \\ \{ywang48, yingying.chen\}@stevens.edu \\ chuah@cse.lehigh.edu
}

\begin{abstract}
Mobile wireless devices (e.g., smartphones, PDAs, and notebooks) play important roles in our daily life, e.g., users often use such devices for bank transactions, keep in touch with friends. Users can also store such information and share with one another via opportunistic peer to peer links. However, peer to peer links are opportunistic links which are intermittent in nature and hence require the store-and-forward feature proposed in Delay Tolerant Networks to provide useful data sharing opportunities. Moreover, due to the limited resources, e.g., communication bandwidth and battery consumption, mobile devices can be selfish and may not be willing to forward data items to other devices that are interested in such items. Hence, effective data dissemination schemes need to be designed to encourage nodes to collaboratively share data. In this paper, we propose a Multi-Receiver IncentiveBased Dissemination (MuRIS) scheme that allows nodes to cooperatively deliver information of interest to one another via chosen delivery paths that utilize few transmissions. Our MuRIS scheme utilizes local historical path and tracks users' interests information maintained by each node. In addition, the charge and reward functions incorporated within our MuRIS scheme stimulate cooperation among nodes such that the nodes have no incentive to launch edge insertion attacks. Furthermore, our charge and reward functions are designed such that the chosen delivery paths mimic efficient multicast tree that results in fewest delivery hops. Extensive simulation studies using real human contact-based mobility traces show that our MuRIS scheme outperforms existing methods in terms of delivery ratio and transmission efficiency.
\end{abstract}

\section{INTRODUCTION}

With the rapid advancement of wireless technologies, mobile wireless devices, e.g., smartphones, PDAs, and laptops have emerged and are gradually woven into our social life. Such devices allow people to access information anywhere at anytime since these devices have increasingly larger storage and support multiple network interfaces including cellular, WLAN, and Bluetooth. Thus, besides using such devices to make phone calls and send text messages, users can utilize these devices to access and store interesting data items such as news clips, sports events, finance forecast, and trending tweets.

While cellular data services are available almost everywhere, constantly using such services to access information is costly because the energy consumed with such constant access is high. On the other hand, it is attractive to exploit peer to peer ad hoc networks [1] formed by these wireless devices utilizing lower-power radios (e.g., Bluetooth or Wi-Fi) to share useful information among users. As such, stored data items can be organized into various categories, e.g., entertainment, finance, politics, technology. Users can acquire the data items

This work is supported in part by the National Science Foundation Grants CNS1016303 and CNS 1016296 from their peers by expressing their interests based on data categories which are used to describe these data items.

Although content dissemination schemes have been proposed for ad hoc networks in the past, e.g. [2], such approaches usually assume that the networks are well-connected. However, interfaces such as $\mathrm{Wi}-\mathrm{Fi}$ and Bluetooth have shorter radio range and hence connectivity between mobile devices using such interfaces is dynamic and intermittent. Delay tolerant networking technology [3] has been proposed to allow nodes in such environments to still communicate by store-and-forward communications. In addition, traditional content dissemination schemes do not consider users' changing interests from time to time. Thus, new content dissemination schemes need to be user-centric and address the intermittent connectivity issue.

Recently, there are active researches in exploring effective schemes for providing content distribution services in Delay Tolerant Networks(DTNs). For example, ContentPlace [4] exploited dynamically learned information about users' social relationships to determine where to place data objects in order to optimize content availability. Similarly, MOPS [5] designed a publish/subscribe system for delay tolerant environments such that nodes within the same community could communicate directly when published data items and interests match while brokers are used to bridge different communities. A cooperative user-centric efficient information dissemination scheme [6] is developed, which used only a few relays and allowed each node to operate distributively. Furthermore, Gao and et. al. [7] proposed a social centrality metric by considering social contact patterns and interests of users simultaneously to achieve efficient content disseminations.

All the above works assumed that users are cooperative and do not refuse to forward data items to others. Such assumptions are not always true in real-world scenarios, since wireless devices have limited resources, e.g., power, storage and available bandwidths provided by opportunistic links. Thus, any useful content dissemination scheme needs to encourage users to cooperate for sharing information with an incentive or reputation mechanism. Toward this end, MobiCent [8] is a credit-based incentive system in DTNs for delivering unicast messages, where its charge/reward functions are designed to encourage users to cooperate and thwart edge insertion and edge hiding attacks. RELICS [9] is another cooperativebased mechanism to combat selfishness in DTNs, in which a rank metric was defined to measure the transit behavior of a node. These two incentive-based schemes focused more on delivering unicast messages, while we are more interested in one-to-many communication pattern, which is typical for 
information sharing in a publish/subscribe system.

The most relevant study to our work is proposed by Ning and et. al. [10], where an incentive based forwarding scheme is developed to reward the last relay node on the delivery path. In this scheme, every node maintains an effective interest contact probability (ECIP) for each data category. Upon encountering each other, two nodes would exchange data messages based on the ECIP to maximize their own expected credit rewards. However, the incentive mechanism rewarding the last-hop relay node is not fair for all other relay nodes. Moreover, the performance of the incentive mechanism degrades when data items are sparsely distributed among nodes due to its overly restrictive replication mechanism.

In this work, we present a Multi-Receiver Incentive-Based Dissemination (MuRIS) scheme that not only encourages nodes to cooperate via our proposed incentive mechanism, but also wisely selects paths that can reach multiple subscribers efficiently. Specifically, we propose multi-receiver based charge and reward functions that would favor the paths which can reach more subscribers at intermediate hops. We further prove that our charge and reward functions can prevent edge insertion attacks by providing no rewarding gain for inserting faked intermediate nodes. Moreover, we show that our information sharing scheme allows nodes to utilize locally maintained information about past node encounters and partial delivery paths to determine if they should forward received data items to other nodes they encounter such that the chosen delivery paths are those that efficiently reach many subscribers.

To evaluate the effectiveness of MuRIS, we used MIT reality with the ONE simulator, which is a trace driven simulator specially designed for DTN environments. The performance of MuRIS is compared with existing data dissemination approaches with or without incentive. The simulation results show that our approach can achieve delivery ratio similar to the Epidemic scheme (where nodes simply re-broadcast whatever they receive) while maintaining a low overhead ratio. Comparing to existing work, MuRIS has a higher delivery ratio with comparable overhead.

The rest of the paper is organized as follows. The models of our scheme is described in Section II. Section III presents our Multi-Receiver Incentive-Based Dissemination (MuRIS) scheme. Section IV evaluates the effectiveness of MuRIS and compares its performance with existing approaches. Finally, Section $\mathrm{V}$ concludes the paper.

\section{A. Network Model}

\section{MODELS}

Each node in the network represents an user who carries a mobile device supporting multiple wireless interfaces including cellular, WLAN, and Bluetooth. The wireless interfaces of all nodes are assumed to be identical, while the rate and duration of contacts between different pairs of nodes are different. In addition, we consider that the message's delivery paths from a source node to destinations may repeat frequently due to the same interests of users and the definite area where the users reside. We assume the nodes in our network are selfish and will not help to relay data unless it can gain some benefits. However, the nodes are authenticated and will not attack the network maliciously. A node is willing to be charged a certain amount of "money" for the data items that it is interested in. Furthermore, each node will be paid a certain amount of "money" for helping to relay data items. We will discuss more about charging and rewarding in Section III.

\section{B. Data Model}

Information in our network may be organized into different categories. For example, news from CNN may be classified into the following categories: politics, weather, entertainments, and etc. All news related to politics can be further described using various sub-categories such as healthcare and debt crisis. A more comprehensive data model based on categories and keywords can be found in [6]. In this work, we use a simplified channel-based model [1], [4], where the information is organized in different channels to which users can subscribe. There are totally $R$ channels in our study.

\section{Publisher/Subscriber (User)}

In our work, each node can be a publisher, a subscriber or both. Each publisher can publish data items that belong to different channels. The set of channels each publisher $j$ publishes is listed as $P C H_{j}=w_{1}, \ldots w_{M}$ where $w_{i}$ is a number between 1 and $M$, and $M$ is the total number of channels that publisher publishes. Further, each subscriber (or a user) has an interest list defined as:

Def 1: An interest list of a user $k$ is a $M \times 1$ vector $I_{k}=$ $\left[i_{k_{1}}, \ldots, i_{k_{M}}\right]^{T}$, where $i_{k_{r}}$ indicates the channel that user $k$ is interested in.

\section{Messages}

There are three types of messages in our system: 1) Probe message is used to record possible paths from publishers to subscribers. They are only forwarded during warmup period or when nodes have been idle for a while. 2) Receipt message is generated by subscribers to confirm the path information carried by probe messages. 3) Data item message is generated by publishers to distribute data contents in the network. In the rest of our work, messages will be used without specification to describe the data item messages.

\section{INCENTIVE DRIVEN INFORMATION SHARING}

In this section, we present the Multi-Receiver IncentiveBased Dissemination (MuRIS) scheme, which aims to provide efficient information sharing in DTNs when non-cooperative users are present. We first provide an overview of MuRIS. Then we introduce the multi-receiver based incentive mechanism used in MuRIS. Next, we define two important concepts, namely the closeness vector, and the feasible path set, before we present our incentive driven information sharing scheme.

\section{A. Overview of Our Incentive Based Forwarding Scheme}

We focus our design on one-to-many dissemination scenarios such as the publish/subscribe systems for DTNs that can benefit from the multicast capability. One simple way to provide one-to-many dissemination is to have all the nodes re-broadcast whatever new data items they receive. However, such scheme results in many useless message replications and hence waste the limited network resources. To address this problem, we propose MuRIS scheme for efficient information sharing, especially for one-to-many dissemination scenarios. 
Our MuRIS scheme dynamically constructs efficient multicast delivery paths for multiple receivers interested in the same data item. In addition, an incentive mechanism is incorporated in MuRIS to encourage selfish intermediate nodes to be cooperative so as to gain some rewards associated with their forwarding efforts. The approach of our incentive based forwarding scheme can be divided into following two parts:

Information Collection: During the warmup period or when have been idle for a while, nodes use probe/receipt messages to learn potential paths from publishers to various subscribers in the network. Additionally, when two nodes encounter, both nodes exchange the path information they collected, and update their knowledge of paths if the other node has a newer or better path. Base on the path information it learns, every node constructs its feasible path set, which will be described more in Section III-D2. Each node in the network records the encounter events, and a closeness vector (defined in Section III-D1) is constructed based on such encounter histories.

Data Forwarding: When two nodes encounter, they first exchange new data items of interests from each other. Then, for the remaining data items at a node, it estimates the potential reward for forwarding any data item based on the closeness vector and feasible path set information, and decides to only forward that data item if the path via the other node can provide the highest expected reward.

\section{B. Background of Generic Incentive Mechanism}

To ease the following discussion, we present here some background on a generic incentive mechanism. In a fair incentive mechanism, all cooperative intermediate nodes along the delivery path of a data item should receive a reward proportional to the resources consumed for the delivery. Moreover, if we assume the identical resource consumption for every intermediate node, the charge and reward regarding a $n$ hop delivery path must satisfy the Equation (1) to ensure the charge can cover all the rewards.

$$
\mathbb{C}(n) \geqslant n \times \mathbb{R}(n),
$$

where $\mathbb{C}(n)$ and $\mathbb{R}(n)$ are the charge to a subscriber and the reward per intermediate node on the delivery path, respectively.

However, an incentive mechanism simply following the Equation (1) is vulnerable to the cheating performed by some greedy intermediate nodes, e.g., edge insertion attacks, to gain additional rewards. Such attacks can be prevented by designing an incentive mechanism that adheres to the following rules [8]:

Rule 1: To prevent an intermediate node from gaining in an edge insertion attack, the reward $\mathbb{R}(n)$ for relaying the data item via an $n$ hop path must satisfy $2 \times \mathbb{R}(n+1) \leq \mathbb{R}(n)$.

Rule 2: To prevent a subscriber from benefiting in an edge insertion attack, the charge $\mathbb{C}(n)$ for receiving a data item via an $n$ hop path must satisfy $\mathbb{C}(n+1)-\mathbb{R}(n+1) \geq \mathbb{C}(n)$.

Although the MDR incentive mechanism in [8] could prevent edge insertion attacks, their work only focused on unicast cases. In this work, our incentive mechanism is designed not only to thwart edge insertion attacks, but also to cooperate our multi-receiver dissemination scheme for DTNs.

\section{Multi-Receiver based Incentive Mechanism}

Here, we first use a simple example as shown in Figure 1 to illustrate the importance of considering collectively the

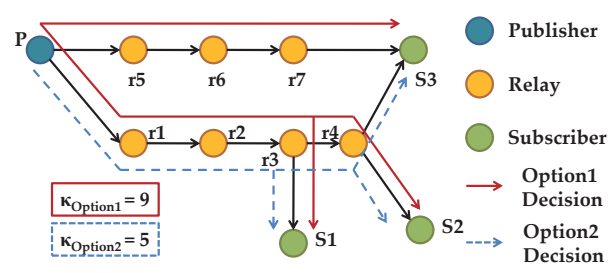

Fig. 1. A comparison of efficiency for different options of path

reachability to multiple receivers in the forwarding decision for one-to-many communication scenarios. All nodes in Figure 1 are mobile nodes which use the same dissemination scheme. Assume that a publisher $P$ wants to send a data item to three subscribers $S 1, S 2$, and $S 3$. Figure 1 shows two forwarding path options: Optionl selects the shortest paths for each individual pub-sub pair, while Option 2 selects a delivery path that minimizes the number of transmissions. It is obvious that Optionl with fewer transmissions is more efficient in energy.

Based on the above observation, we aim to design an incentive mechanism that encourages nodes to cooperate such that the selected forwarding paths use few transmissions and hence achieves high network efficiency. Furthermore, we realized that to encourage nodes to make such forwarding decisions in one-to-many dissemination scenarios, the delivery status of messages must be considered. Therefore, we assume that every message has a header which contains a pair of values $(R S S$, $R N S$ ) defined as follows:

Def 2: The Reachable Subscriber Size (RSS) of a data item $m$ is the number of subscribers that have already received $m$ on the path that this data item has been through.

Def 3: The Relay Node Size (RNS) of a data item $m$ is the number of intermediate nodes that have successfully delivered at least one copy of $m$ to a subscriber.

With above two parameters, we propose our multi-receiver based charge and reward functions:

$$
\begin{gathered}
\mathbb{C}\left(n, \nu_{m}\right)=2^{N}-2^{N-n}\left(1+\theta\left(n, \nu_{m}\right)\right) \\
\mathbb{R}\left(n, \nu_{m}, \psi_{m}\right)=2^{N-n}\left(1-\phi\left(\nu_{m}, \psi_{m}\right)\right),
\end{gathered}
$$

where $\nu_{m}, \psi_{m}$ are values of $R S S$ and $R N S$ respectively, and $\theta(),. \phi($.$) are two synthetic functions that are introduced$ to utilize the information from $R S S$ and $R N S$. The RSS and RNS do not require the prior knowledge of the topology because they are locally derived from the relaying experience of each relay node. Instead, the RSS and RNS collect the topology information dynamically during relay process, which is particularly suitable for ad-hoc networks. We aim to design the charge and reward functions in such a way that can favor efficient delivery paths traversing more subscribers. To demonstrate the challenges, we summarize the conditions that our incentive mechanism must satisfy as follows:

1) Both charge and reward functions must be positive.

2) Equation (1) must be satisfied to ensure the charge can cover all rewards.

3) Rule 1 must be satisfied to ensure no intermediate node can launch an edge insertion attack.

4) Rule 2 must be satisfied to ensure no subscriber can launch an edge insertion attack.

We particularly choose the function described in Equation (4) 
to be our $\theta\left(n, \nu_{m}\right)$ to ensure the condition (1), (2), and (4).

$$
\theta\left(n, \nu_{m}\right)= \begin{cases}0 & \text { if } \tau=0 \\ \frac{\nu_{m}}{\tau} & \text { if } 1<\nu_{m} \leq \tau,\end{cases}
$$

where $\tau=\max \left(\nu_{m}\right)$ is a constant in the system and $\nu_{m}$ is maintained locally by every node itself.

Similarly, to guarantee condition (1), (2), and (3), we define $\Lambda=\nu_{m}+\psi_{m}$ and $\tau^{\prime}=\max (\Lambda)$, and choose the function described in Equation (5) to be our $\phi\left(\nu_{m}, \psi_{m}\right)$.

$$
\phi\left(\nu_{m}, \psi_{m}\right)= \begin{cases}0 & \text { if } \Lambda=0 \\ \log _{\tau^{\prime}}(\Lambda) & \text { if } 1 \leq \Lambda \leq \tau^{\prime},\end{cases}
$$

With the chosen functions $\theta($.$) and \phi($.$) , our charge function$ eventually favors the paths with fewer hops and larger RSS values, and our reward function favors delivery paths with fewer hops and smaller RSS values. This design is rational, since selfish intermediate nodes would favor the path that can reach more potential subscribers to maximize its rewards in total. Moreover, our proposed charge and reward functions can be shown to be incentive compatible under edge insertion attacks with the proofs in our technical report [11].

\section{Closeness Vector and Feasible Path}

MuRIS exploits historical encounters and path traversal information available at each individual node to assist incentivebased forwarding. We introduce two building blocks, closeness vector and feasible path set that can be used to aid in making forwarding decision. Particularly, closeness vector is a metric used to predict future encounters, and feasible path set provides possible paths information for data forwarding.

1) Closeness Vector: The encounter time and the associated contact duration recorded by a node when it meets another node can be used to predict future encounters. We define the concept of closeness using the cumulative window (Cwindow) approach [12], which calculates the average node encounter duration during previous time windows. The node $N_{i}$ maintains its list of encountered nodes in the most recent observation window of size $W$ with $W=\rho \times \Delta t$, where $\Delta t$ is the unit time, and $\rho$ is the number of units within the observation window. The Closeness between two nodes $N_{i}$ and $N_{j}$ is defined as: $c_{i j}=\frac{\sum_{k=1}^{\rho} \delta_{i j} \times \Delta t}{W}$, where $\delta_{i j}$ is an indicator function, with a value of 1 when $N_{i}$ and $N_{j}$ encounters during a unit time slot $\Delta t$, and 0 otherwise.

Thus, each node maintains a closeness vector:

Def 4: A node $N_{i}$ 's Closeness Vector $C_{i}$ is a $J \times 1$ vector with: $C_{i}=\left[c_{i 1}, \ldots, c_{i J}\right]^{T}$, where $J$ is number of nodes that $N_{i}$ has encountered during the previous time window.

We note that each node can compute its Closeness Vector based on its own local information.

2) Feasible Path Set: Since nodes move around, the available paths between a node pair change dynamically. A common way to describe feasible paths in DTNs is to use a sequence of nodes and their corresponding probabilities of reaching a certain destination [5], [7]. However, such probabilities may not capture the dynamics of the actual node movements since they are often computed based on the assumptions that the node inter-encounter times are exponentially distributed. In this work, we let each node maintain a set of paths that have been used in the past to reach certain subscribers. We refer to this set of paths as the feasible path set.
Def 5: Feasible Path Set (FPS) of node $N_{i}$ is defined as $\mathbb{F}_{i}=\left(\mathbb{V}_{i}, \mathbb{Q}_{i}\right)$, where $\mathbb{V}_{i}=\left\{V_{i}^{1}, \ldots, V_{i}^{M}\right\}$ where $V_{i}^{m}=$ $\left\{v_{m, 1}, \ldots, v_{m, S}\right\}$, is a set of $S$ subscribers $\left(v_{m, j}\right)$ interested in channel $m$ that node $N_{i}$ is aware of; $\mathbb{Q}_{i}=\left\{Q_{i}^{1}, \ldots, Q_{i}^{M}\right\}$ where $Q_{i}^{m}=\left\{p_{m, 1}, . . p_{m, K}\right\}$, is a set which contains $K$ paths that have been used to reach the subscribers in the corresponding set of subscribers, i.e. $V_{i}^{m}$.

Construction Process: To collect historical path information initially, every publisher regularly sends a probe message labeled as a particular data category that this publisher will publish. Nodes only forward the probe message during the warmup period, or when nodes are idle for a while. Whenever the probe message reaches a subscriber interested in it, the subscriber sends a receipt message containing the path information carried by the corresponding probe message to all relay nodes on the recorded path. Eventually, every involved node can receive the historical path information, and construct or update its FPS for corresponding data category.

To cope with the highly dynamic environment of DTNs, in addition to construct or update the FPS by means of probe/receipt messages, every node exchanges its FPS with other nodes it encounters to keep the path information updated. By comparing its FPS with the other node's (remote FPS), a node can can learn new or better (fewer hops) paths to reach any of the subscribers.

Control of feasible path sets: We also use two parameters to control the size of the feasible path set: $N$ (maximum hopcount) and $L_{p}$ (path's lifetime). Any feasible path is eliminated if its hopcount reaches $\mathrm{N}$ or its lifetime expires. Considering that the behaviors of nodes in DTNs may mimic mobile social networks, in our study, we set $N=6$ for the MIT-trace based on the average separation for humans discussed in [13]. Furthermore, we set $L_{p}=48$ hours for the MIT-trace. Since the lifetime of paths affects the correctness of the feasible paths, we will study the impact of the lifetimes of feasible paths in the near future.

\section{E. Incentive Driven Information Sharing}

A node may be aware of multiple paths that can reach different number of subscribers. Thus, when a node encounters another node, it needs to decide if it should forward a data item to that node based on the expected reward it can gain. We calculate the expected reward using the Closeness Vector and the reward function described in Section III-C.

1) Expected Rewards: We first discuss how expected reward is computed. Assume that a message has gone through $n$ hops when $N_{i}$ receives it.

Def 6: The expected reward for node $N_{i}$ via the next hop node $N_{j}$ is the estimated reward that $N_{i}$ can gain if the data item is successfully delivered to subscribers via the paths starting from $N_{j}$ before the message expires. Assume that there will be $K_{l}$ more subscribers that can receive the message at $l^{\text {th }}$ hop after $N_{i}$. Therefore, $K_{0}=\nu_{m}$ and $K_{1}$ is the number of subscribers that receive the message from $N_{j}$. The expected reward can be computed as follow:

$$
\mathbb{E}_{i j}=\sum_{l=1}^{P} K_{l} \times 2^{c_{i j}} \times \mathbb{R}\left(n+l, \nu_{m}+K_{l-1}, \psi_{m}+M_{l}\right),
$$

where $P=(N-n)$ is the number of additional hops allowable 
before the maximum hop count is reached, and $M_{l}$ is a cumulative function defined as: $M_{l}=\sum_{n=1}^{l} \delta_{n}$, where $\delta_{n}$ is an indicator with a value of 1 if $K_{n} \neq 0$ and 0 otherwise.

Equation (6) is the total expected reward $N_{i}$ can receive after forwarding the message to $N_{j}$. However, $K_{l}$ in Equation (6) requires complete path information which is not available at node $N_{i}$. Therefore, we simplify the problem as follows: assume that currently node $N_{i}$ knows of $d$ paths to a subscriber $S_{j}$ via a next-hop node $N_{j}$ with each path having to traverse an additional $w_{k}, k=1, \ldots, d$ hops after node $N_{i}$. We use $h_{l}=\sum_{k} w_{k} / d$ to estimate the future hopcounts to $S_{j}$, and assume there is no change in $\nu_{m}$ and $\psi_{m}$. The expected reward of any data item message can then be computed as:

$$
\mathbb{E}_{i j}=\sum_{l=1}^{D} 2^{c_{i j}} \times \mathbb{R}\left(n+h_{l}, \nu_{m}, \psi_{m}\right),
$$

where $D$ is the number of possible subscribers, both of them can be derived from $\mathbb{V}_{i}$ and $\mathbb{Q}_{i}$ in $N_{i}$ 's Feasible Path set.

2) Dissemination Scheme: Our multicast-efficient incentive compatible forwarding scheme is described in Algorithm 1. In our scheme, a node always firstly tries to transmit any message carrying a data item to any node who has interest in the message. Further, we denote Feasible Next Hop Set of a message as nexthopList $(m)$ in Algorithm 1, which are the next-hop nodes of all feasible paths to any subscriber of the message currently present in node $N_{i}$ 's Feasible Path Set. For each node in the Feasible Next Hop Set, node $N_{i}$ will compute its expected reward by using the Equation (7), and see which next-hop node (say Node $N_{j}$ ) yields the maximum reward. If this maximum reward for forwarding to $N_{j}$ is larger than that without forwarding, and $N_{j}$ is within the communication range of $N_{i}$, then that message will be forwarded.

To guarantee deliveries and limit overhead, only a publisher is allowed to forward its own message to any node that it encounters when there is no next-hop node in its feasible next hop set of the message.

\section{EVALUATION}

We evaluate our proposed multicast efficient incentive driven forwarding scheme using the Opportunistic Network Environment (ONE) simulator [14], which is a trace driven simulator specially designed for DTN environment. In this section, we first describe our simulation methodology, followed by the comparison and discussions.

\section{A. Simulation Methodology}

To show the generality effectiveness of MuRIS, we choose the human contact-based trace, which is from the MIT reality mining experiment [15]. The MIT-trace is collected from smart phones carried by 97 participants at MIT campus over a 6-month period. Each trace contains information about the IDs of the devices within the communication range of each other, and the starting and ending times of their encounters. We use the first 8 days of the traces for our simulation. In particular, we use the first day trace as the data for our warmup period, where nodes build the knowledge of subscribers and construct feasible path sets for our dissemination scheme. The rest of the 7-day trace is used for the evaluations of different dissemination schemes. We compare MuRIS with three existing schemes, namely Epidemic scheme [16], RELICS scheme [9], and Incentive forwarding scheme [10].

\section{B. Experiment Setup}

We use the following default settings for our simulation studies: there are three publishers in the network, each publishing data items belonging to a particular data channel. The interarrival time of a new data item is uniformly distributed in the range $[140 s, 180 s)$. Each publisher starts generating data items after the warmup period, and stops generating after 1000 items have been published. We set the storage size of each node to be $100 \mathrm{M}$ bytes (which can store $70 \mathrm{~K}$ data items, each with an average size of $2.5 \mathrm{~K}$ bytes) so that there will be no data item losses due to limited storage size. Thus, we can focus our study merely on the efficiency of the different dissemination schemes. We vary the number of subscribers for each data channel to study its impacts on the delivery performance.

The following metrics are used to compare different dissemination schemes: Delivery Ratio: The proportion of messages that have been delivered out of the total unique messages created. Average Delay: The average time that is used to deliver messages to corresponding subscribers. We consider the delay time of undelivered messages as the time that they have been staying in the network by the time our simulation stops. Overhead Ratio: The ratio of the total number of messages relayed over the total number of unique messages delivered.

\section{Performance Comparison}

1) Effectiveness of MuRIS: According to [5], [17], the nodes within the MIT trace form different communities. Thus, we select two scenarios to study the impact of having publishers/subscribers coming from the same or different communities. In the Scenario 1, we select both the publisher and subscribers of a particular data channel from the same community, while in the Scenario 2, the publisher and subscribers come from different communities. We present simulation results for the cases where the message expiration time is set to 1 day

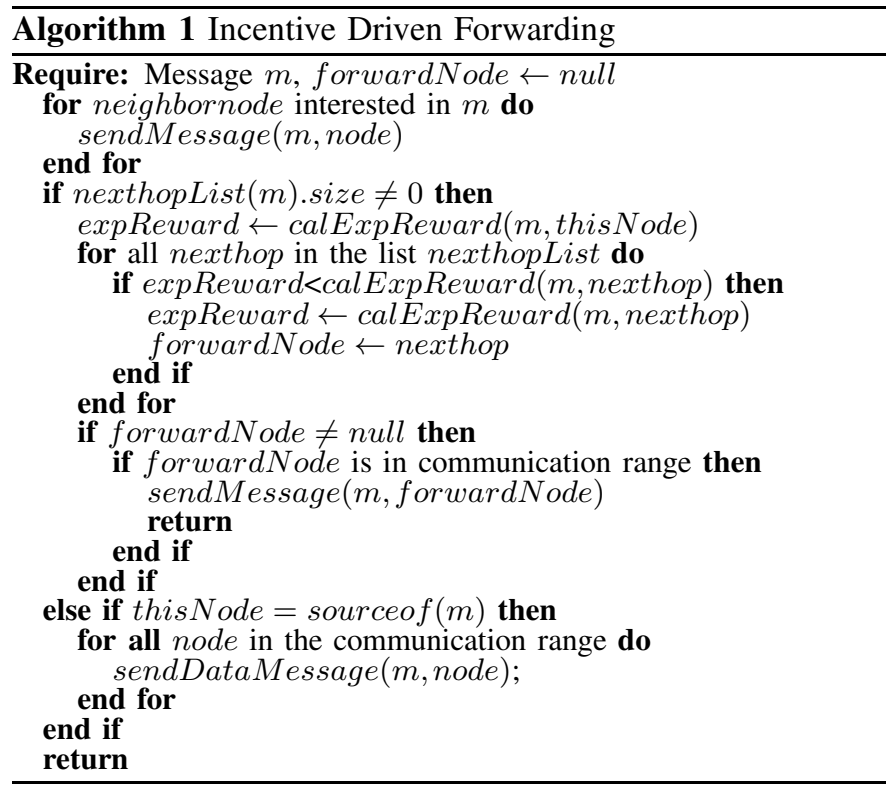




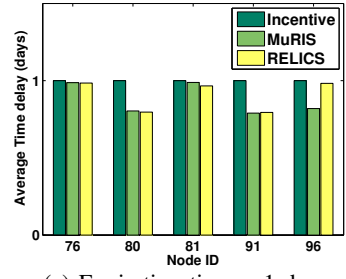

(a) Expiration time $=1$ days 3 subscribers per channel, Scenario 2

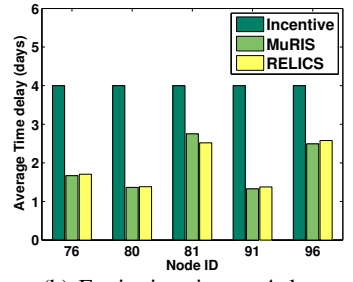

(b) Expiration time $=4$ days

3 subscribers per channel, Scenario 2

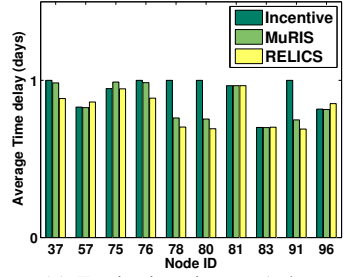

(c) Expiration time $=1$ days

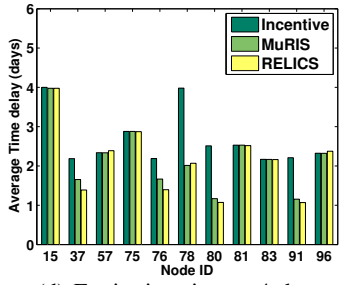

(d) Expiration time $=4$ days

6 subscribers per channel, Scenario

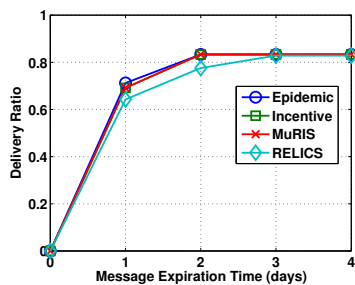

(a) 6 subscribers per channel for Scenario 1

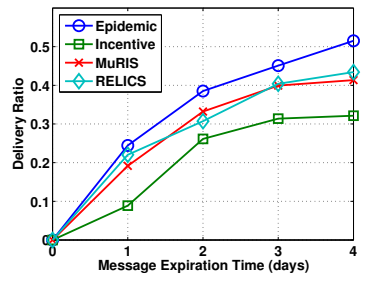

(c) 6 subscribers per channel for Scenario 2

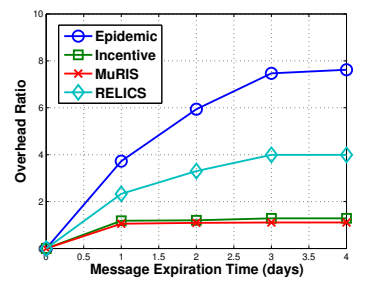

(b) 6 subscribers per channel for Scenario 1

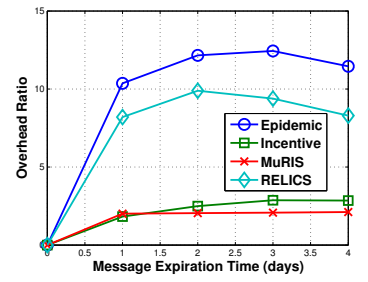

(d) 6 subscribers per channel for Scenario 2

Fig. 2. Delivery ratio and overhead ratio with MIT-trace for two scenarios

through 4 days in Figure 2. The publisher and subscribers from the same communities encounter one another frequently and hence we expect to see better delivery performance. From Figure 2(a) we see that all four schemes achieve very similar delivery ratio for Scenario 1. Figure 2(b) shows that MuRIS has the lowest overhead ratio while achieving a comparable high delivery ratio among all four schemes. The Epidemic scheme has the highest overhead ratio due to its blind replication strategy. The overhead ratio of RELICS is less than the Epidemic scheme's, but is still much higher than the Incentive and MuRIS. By using the feasible path set, MuRIS is aware of multicast-efficient paths to reach subscribers, thus the number of copies of a data item in the network is minimized. Compare the results in Figure 2, we observe that MuRIS works well for both scenarios, while the Incentive scheme has lower delivery ratio and RELICS scheme has higher overhead ratio when publishers and subscribers are from different communities.

2) Latency Efficiency: We also studied the average delivery latency of the four schemes for the scenarios with different subscribers. Figure 3 presents the distribution of the average delay when the data item expiration time is 1 day and 4 days. We can observe that MuRIS has much lower average delay for most subscribers than the Incentive scheme does. This indicates that MuRIS chooses the appropriate delivery paths that can reach subscribers faster and yet with fewer total transmissions in terms of the overhead ratio illustrated in Figure 2(d). The Incentive scheme has much longer average delay as a result of its overly restrictive replication strategy. MuRIS has a similar average delay as RELICS scheme does,

while we can find the overhead ratio of MuRIS is much lower in Figure 2(d). We didn't include the results of Epidemic scheme in Figure 3 because Epidemic scheme can always achieve the shortest delay due to its blindly forwarding nature.

\section{CONCLUSIONS}

In this work, we have proposed an incentive driven dissemination scheme called MuRIS that not only encourages nodes to cooperate but chooses delivery paths that can reach as many subscribers as possible with fewest transmissions. The wise choice of delivery paths is achieved via our proposed multi-receiver based incentive mechanism. Furthermore, our charge and reward functions not only thwart edge insert attacks but also allows us to achieve high network efficiency. MuRIS exploits locally maintained node encounter history and historical path information to construct closeness vector and feasible path set. Simulation studies using human-contact based traces show that MuRIS outperforms other existing schemes in achieving high delivery ratio with low overhead ratio. MuRIS performs especially well when the publisher and subscribers come from different communities.

\section{REFERENCES}

[1] V. Lenders, G. Karlsson, and M. May, "Wireless ad hoc podcasting," in ACM SigMobile Mobile Computing and Communications Review, 2008.

[2] L. Yin and G. Cao, "Supporting cooperative caching in ad hoc networks," Mobile Computing, IEEE Transactions on, Jan. 2006.

[3] K. Fall, "A delay-tolerant network architecture for challenged internets," ser. SIGCOMM '03.

[4] C. Boldrini, M. Conti, and A. Passarella, "Contentplace: Social-aware data dissemination in opportunistic networks," in MSWiM'08, 2008.

[5] F. Li and J. Wu, "Mops: Providing content-based service in disruptiontolerant networks," ser. ICDCS '09.

[6] M. Chuah, P. Yang, and P. Hui, "Cooperative user centric information dissemination in human content-based networks." in ICPADS'10, 2010.

[7] W. Gao and G. Cao, "User-centric data dissemination in disruption tolerant networks," in INFOCOM '11, 2011.

[8] B. B. Chen and M. C. Chan, "Mobicent: a credit-based incentive system for disruption tolerant network," ser. INFOCOM'10.

[9] M. Uddin, B. Godfrey, and T. Abdelzaher, "Relics: In-network realization of incentives to combat selfishness in dtns."

[10] T. Ning, Z. Yang, X. Xie, and H. Wu, "Incentive-aware data dissemination in delay-tolerant mobile networks," in SECON '11, 2011.

[11] Y. Want, M. Chuah, and Y. Chen, "Incentive Driven Dissemination Scheme in Delay Tolerant Networks," Stevens Institute of Technology, Tech. Rep., Jan 2012.

[12] P. Hui, J. Crowcroft, and E. Yoneki, "Bubble rap: Social-based forwarding in delay tolerant networks," Mobile Computing, IEEE Transactions on, vol. PP, no. 99, 2010.

[13] J. Guare, Six degrees of separation : a play. Vintage Books, 1990.

[14] A. Keränen, J. Ott, and T. Kärkkäinen, "The one simulator for dtn protocol evaluation," ser. Simutools '09.

[15] N. Eagle and A. S. Pentland, "CRAWDAD trace set mit / reality / blueaware (v. 2005-07-01)," 2005.

[16] A. Vahdat and D. Becker, "Epidemic Routing for Partially Connected Ad Hoc Networks," Duke University, Tech. Rep., apr 2000.

[17] P. Hui, E. Yoneki, S. Y. Chan, and J. Crowcroft, "Distributed community detection in delay tolerant networks," ser. MobiArch '07. 